\title{
Desarrollo de comunidades de aprendizaje en línea para titulaciones jurídicas. \#Relaciónate: plan transversal de innovación docente a través del uso de nuevas tecnologías en el Grado en Relaciones Laborales ${ }^{1}$
}

\section{Marina Revuelta García ${ }^{a}$ y Araya Alicia Estancona Pérez}

${ }^{a}$ Profesora Ayudante Doctor de Derecho del Trabajo y de la Seguridad Social. Departamento de Derecho Privado. Universidad de Cantabria (marina.revuelta@unican.es), ${ }^{b}$ Profesora Ayudante Doctor de Derecho Civil. Departamento de Derecho Privado. Universidad de Cantabria (araya.estancona@unican.es).

\begin{abstract}
This paper shows the results of a teachinginnovation Project called \#Relaciónate of the University of Cantabria. This Project was designed to stablished a communication and specialist information channel between Labour Relations Degree students and teachers. The regular publication through Twitter was focused on legal reports, sentences, doctrinal analysis selected by teachers and specialised profiling network-like official bodies, professionals, teachers connected by social network, ecc.-. This particular system creates an online learning community which allows us to contextualize and share theoretical contents being taught in classroom and to improve the students involvement in learning processes and their relevant skills and values, such as self-directed learning, critical thinking or information management.
\end{abstract}

Keywords: Online learning community, self-directed learning, legal science, ICT and social network, university teaching, Twitter.

\section{Resumen}

Se presentan los resultados del proyecto de innovación docente \#Relaciónate, concebido para dotar a los estudiantes y profesores del Grado en Relaciones Laborales de un canal de comunicación y difusión de información especializada en las diversas materias que integran el plan de estudios de la titulación. La publicación periódica a través de Twitter de noticias jurídicas, sentencias o análisis doctrinales seleccionados por los diferentes profesores del Grado, junto a la configuración de una red de perfiles de referencia organismos oficiales, editoriales jurídicas, profesionales del sector, profesores activos en redes sociales, etc.-, se traduce en la creación de una comunidad de aprendizaje en linea que permite la contextualización de los contenidos teóricos impartidos en el aula, favorece la implicación de los estudiantes en el proceso de aprendizaje y contribuye a la mejora de competencias, habilidades y valores esenciales para los estudiantes, tales como el aprendizaje autónomo, el razonamiento crítico o la capacidad de gestión de información.

Palabras clave: Comunidad de aprendizaje en línea. TIC y redes sociales aplicadas a la docencia universitaria. Twitter.

\footnotetext{
${ }^{1}$ Este trabajo se ha desarrollado en el marco del Proyecto de Innovación Docente "\#RELACIÓNATE: Plan transversal de innovación docente a través del uso de nuevas tecnologías en el Grado en Relaciones Laborales" de la IV Convocatoria de Proyectos de Innovación Docente de la Universidad de Cantabria, gracias a la colaboración de la profesora Lourdes López Cumbre, Catedrática de Derecho del Trabajo y de la Seguridad Social de la Universidad de Cantabria. Y sus conclusiones son fruto, asimismo, de la investigación realizada en la Leicester Law School, con la asistencia de la profesora Maribel Canto-López, Senior Fellow of the Higher Education. Lecturer in Law. University of Leicester.
} 


\section{Introducción}

Los New Millennium Learners (NML) demandan medios digitales en los sistemas educativos: alfabetización digital, permanente necesidad de estar conectados, inmediatez y multitarea, carácter social o aprendizaje experimental (Gisbert, M., y Esteve, F., 2011, p. 52). Como complemento de las metodologías tradicionales de enseñanza, han adquirido protagonismo las herramientas online: diversas plataformas virtuales, como Moodle, que facilitan el desarrollo de docencia virtual, la difusión de contenidos complementarios a las clases presenciales, la realización de tareas de seguimiento e, incluso, actividades colaborativas en línea a modo de workshops o foros de debate. Pero no dejan de surgir nuevas opciones: publicación de vídeos, podcasts, blogs, herramientas de gamificación, glosarios o wikis (Holgado Sáez, C., 2011, pp. 97-103), con mención especial de Google for Education (Laro González, E., 2020. pp. 15-17).

El Espacio Europeo de Educación Superior (EEES) require la adaptación de la docencia universitaria a tres principios esenciales: a) una mayor implicación y autonomía del estudiante, b) el empleo de metodologías de enseñanza más activas y c) un nuevo papel del profesorado como agente creador de entornos de aprendizaje que estimulen a los alumnus (Alegre Nueno, M., Esteve Segarra, A., López Balaguer, M., y Taléns Visconti, E., 2018, p. 112). El objetivo básico es que el alumno "aprenda a aprender", garantizando que haya aprehendido conocimientos y haya aprendido a manejarlos (Blanco García, A.I. 2016, p. 29). Y se formenta la adquisición de las habilidades y competencias demandadas por las empresas, que facilitarán la empleabilidad de los estudiantes: el trabajo en equipo par la mejora en su capacidad de interrelacionarse con sus compañeros, la promoción de su independencia frente a los docentes, la exposición de otras ideas y vías de aprendizaje compartido y colaborativo (Cantó-López, M., 2020, pp. 95-107).

En este proceso, todo mecanismo que facilite el trabajo autónomo del estudiante resulta útil. De este modo, las tecnologías de la información y la comunicación (TICs) adquieren un papel esencial para eliminar espacio-temporales de la enseñanza y el aprendizaje; b) conseguir un mimetismo de la Universidad con la sociedad de las TIC's. (Quintero Lima, M.G., 2012, p. 17), por lo que ayudan a conectar con los estudiantes y a fomentar su implicación en el proceso de aprendizaje, mitigando la cadena que los docentes observamos habitualmente y por la que si aburrimos no hay interés y si no hay interés no hay resultados (Fernández-Coca, A., 2011, p. 76).

En este contexto, destaca el éxito del concepto de las redes sociales (Vázquez-Martínez, A.I., y CaberoAlmenara, J., 2015, pp. 255-256.), con las que se busca aprovechar la "hiperconectividad" de los jóvenes en beneficio de su aprendizaje y evitar el desfase generacional con los estudiantes (Gómez, M., Roses, S., y Farias, P., 2012, p. 136). En comparación con otras -como Facebook, Instagram, o YouTube-, Twitter presenta una perfil eminentemente informativo y menos lúdico que el resto permitiendo una comunicación horizontal en tiempo para una transmisión más ágil de contenidos de interés y la creación de nuevos contactos (Periago Morant, J.J., 2019, p. 78). Por ello es considerada la herramienta idónea como recurso complementario en la educación superior (Vázquez-Martínez, A.I., y Cabero-Almenara, J., 2015, p. 265.), facilitando la rápida difusión de información de actualidad que permita contextualizar y profundizar en los contenidos impartidos en el aula (Arrabal Platero, P., 2019, p. 108).

En consecuencia con lo indicado, el proyecto de innovación docente \#Relaciónate, cuyos resultados se presentan en esta comunicación, fue concebido para dotar a los estudiantes y profesores del Grado en Relaciones Laborales de la Universidad de Cantabria de un canal de comunicación y difusión de información especializada en las diversas materias que integran el plan de estudios. Inspirado en experiencias previas del uso de Twitter y en el movimiento \#Tweacher (Romero López, S., 2016, pp. 8085) en asignaturas de planes de estudios de titulaciones jurídicas, por la solvencia de su ejecución y los 
destacables resultados obtenidos por los profesores responsables de los mismos (Nieto Rojas, P., 2017, pp. 51-66 y Alegre Nueno, M., Esteve Segarra, A., López Balaguer, M., y Taléns Visconti, E., 2018, pp. 116117), este plan de innovación docente surge como medida orientada a la consecución de resultados de una de las acciones de mejora incluida en el Informe Final del Sistema de Garantía Interno de la Facultad de Derecho de la Universidad de Cantabria del año 2018, relativa al fomento de la oferta de actividades formativas complementarias y a procurar su mayor visibilidad. Sobre la base de un proyecto desarrollado, se estiman como oportunidades: a) la motivación de los estudiantes sobre las redes sociales es importante; b) para los estudiantes, las aplicaciones son intuitivas; c) fomentan el respeto, la tolerancia, la igualdad, la valoración por el trabajo de los demás; y d) permiten romper con el esquema tradicional de clases, atribuyendo un contenido innovador que motivará al alumnado, haciendo el aula más enriquecedora (Martínez, A., Fernández, R., 2017, p. 184.). Con el objetivo prioritario de contribuir a la mejora de la calidad de la docencia y de la formación académica del Grado en Relaciones Laborales, se caracteriza por su carácter transversal, dirigido a que todos los alumnos y profesores de esta titulación puedan participar proponiendo temas de interés, accediendo a los recursos publicados, aportando su opinión, conocimientos y experiencias, lográndose así importantes sinergias.

\section{Objetivos}

El trabajo tiene por objeto la presentación de un plan de innovación docente como programa piloto introducido en el Grado en Relaciones Laborales de la Universidad de Cantabria a través de la difusión de contenidos y materiales docentes de Calidad para el studio del Deercho del Trabajo y de la Seguridad Social en la red social Twitter. La innovación introducida plantea una programación de la asignatura atractiva y motivadora para el alumnado, que se sentirá involucrado con la organización docentes a través de un papel activo en un entorno virtual.

A través de la creación de un espacio novedoso en el ámbito universitario, a lo largo de este artículo expondremos la medología, cronograma, plan de trabajo, contenidos y resultados del aprendizaje que servirán de preparación a los estudiantes para afrontar los retos derivados del aumento de la complejidad de las relaciones laborales y su marco normativo. En particular, se potenciará su capacidad de aprendizaje autónomo e implicación en el proceso de formación académica, el desarrollo de un razonamiento crítico y creativo, ejercitando las habilidades para debatir y argumentar con compañeros y profesores; se fomentará la organización y gestión de la información así como el trabajo en equipo y colaboración en la consecución de objetivos comunes, mejorando la cohesión del grupo y el aprendizaje colaborativo.

\section{Desarrollo de la innovación}

La ejecución de este proyecto de innovación docente comenzó en el curso 2018/2019, dedicando una primera sesión a dar a conocer entre los estudiantes la actividad propuesta, de carácter voluntario, incidiendo en los aspectos necesarios para el uso apropiado de la plataforma Twitter y de las herramientas que esta ofrece: configuración de cuenta personal y perfil específico para la actividad; cómo twittear incluyendo textos, fotos, vídeos o enlaces web; uso de etiquetas comunes que permitan encontrar las publicaciones; retwittear con y sin contenido; realizar búsquedas de tweets, cuentas, temas o conversaciones; crear una red de contactos especializados y fiables -instituciones oficiales, profesores, medios de comunicación, editoriales jurídicas- que sirva para comunicarse, intercambiar información y actualizar contenidos; mostrar interés por temas y publicaciones a través de la pestaña "me gusta"; comentar las publicaciones de otros y las propias; aludir a otros participantes; enviar comentarios 
privados, etc. En esa primera sesión se destacó, por encima de todo, la importancia de la política de seguridad y privacidad para un uso responsible de la red social.

Desde entonces, la actividad de la cuenta principal (@RelaciónateUC) ha sido intensa, realizando numerosas publicaciones sobre las materias vinculadas al temario de las asignaturas de las titulaciones de Grado en Relaciones Laborales y Derecho, que han servido de apoyo y complemento para la realización de actividades teórico-prácticas en el aula, para profundizar sobre aspectos de mayor complejidad, para argumentar en los debates propuestos y conformar una opinión crítica, para mantenerse al día de las incesantes novedades normativas que se han producido durante estos últimos meses en materia laboral y de protección social, así como de los criterios jurisprudenciales más novedosos.

A estos efectos, dado que se trata de un plan trasversal en el se encuentran directamente implicadas numerosas asignaturas vinculadas al área de Derecho del Trabajo y de la Seguridad Social, fundamentalmente del Grado en Relaciones Laborales (Derecho del Trabajo, Derecho Sindical, Seguridad Social, Seguridad en el Trabajo y Acción Social en la Empresa, Derecho Sancionador Social, Políticas Sociolaborales de Empleo y Género, Estrategias de Negociación Colectiva, Gobierno Corporativo y Responsabilidad Social), y también dos asignatura del Grado en Derecho (Derecho del Trabajo y Protección Social), ha resultado esencial la participación de los profesores implicados, tanto de aquellos dedicados a tiempo completo a la docencia como, también, de un importante grupo de profesores asociados que, como profesionales de reconocido prestigio en el ámbito de las relaciones laborales abogados laboralistas, magistrados de la jurisdicción social, especialistas en Seguridad Social, técnicos de recursos humanos, expertos en prevención de riesgos laborales, etc.-, han aportado sus conocimientos y experiencia a través de las redes sociales. Labor, esta, que ha requerido un importante esfuerzo de coordinación para seleccionar los contenidos al ritmo de las explicaciones realizadas en las diferentes asignaturas de la titulación.

La respuesta de los estudiantes ha sido muy positiva: han mostrado interés por las publicaciones a través de la pestaña "me gusta"; han realizado menciones a la cuenta principal del proyecto; han publicado mensajes al hilo de los contenidos twitteados en la cuenta principal; han compartido utilizando las etiquetas vinculadas al proyecto, etc. Sin duda, el aspecto más destacable ha sido el aumento de su participación en el aula, fundamentalmente realizando valoraciones sobre contenidos publicados en Twitter, demostrando, con carácter general, un alto grado de implicación e interés por la propuesta. Como actividad voluntaria, muchos alumnos han creado una cuenta y siguen el perfil @RelaciónateUC. Pero, incluso aquellos otros que han optado por no participar activamente en la red social, con carácter general, se mantienen más informados y realizan intervenciones orales en el aula aludiendo a los contenidos difundidos.

Por último, respecto al seguimiento del desarrollo del proyecto, este se ha realizado a través de dos vías fundamentales. En primer lugar, con el control de la participación de los estudiantes en Twitter, a cuyos efectos la aplicación dispone de una herramienta que ofrece información sobre la actividad del panel que muestra los datos sobre la repercusión en la audiencia (interacciones, detalle sobre tweets, retweets, respuestas, "me gusta", seguimientos, etc.) y permite descargar las métricas al gestor de la cuenta. En segundo lugar, incorporando en las clases teóricas y prácticas cuestiones que han sido trabajadas en la plataforma digital, para poder valorar los conocimientos adquiridos por los alumnos con su participación en la actividad propuesta. Como muestra de lo indicado, el planteamiento de algunos de los supuestos prácticos evaluables que integraban la evaluación continua de algunas asignaturas se ha basado en cuestiones previamente publicadas en Twitter, de modo que quienes han aprovechado los recursos disponibles en la plataforma han obtenido resultados académicos óptimos. 


\section{Resultados del proyecto}

\subsection{Percepción del profesorado implicado}

La metodología utilizada en este proyecto permite que los estudiantes seleccionen contenidos que resultan de su interés y que, gracias a su publicación en Twitter, conduzcan en cierta medida el desarrollo de las clases teóricas y, sobre todo, de las prácticas, advirtiéndose una mayor implicación en las materias tratadas, más colaboración entre ellos y mucha más participación mediante intervenciones orales durante el desarrollo de las sesiones en el aula. A modo de ejemplo, una alumna publicó una noticia sobre la negociación colectiva en el trabajo en plataformas digitales, que fue objeto de análisis en una clase práctica y que suscitó un interesante debate entre los asistentes (resultado núm. 1).

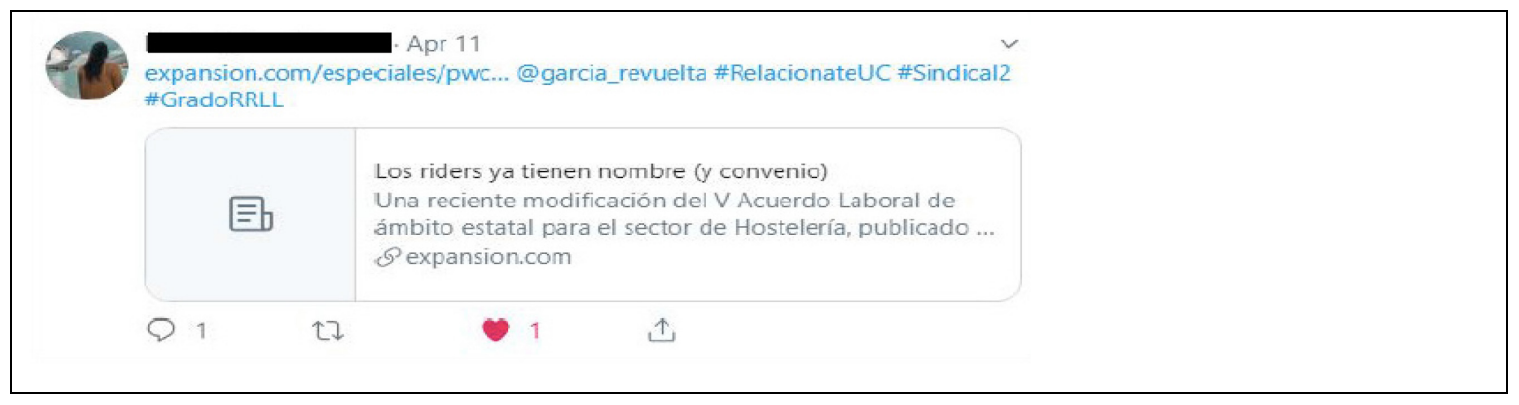

Resultado núm. 1: Publicación de alumna sobre tema de debate en el aula.

Se ha producido una mejora de la participación activa de los estudiantes implicados, tanto en entornos virtuales como, gracias a ellos, en el aula. En concreto, la plataforma Twitter les ha permitido: a) mostrar interés por las publicaciones de la cuenta vehicular del proyecto (RelaciónateUC) pulsando en la pestaña "me gusta", representada con un corazón, de manera que los tweets quedaran registrados en sus perfiles para facilitar su posterior consulta (resultado núm. 2); b) twittear o retwittear información de actualidad (noticias, sentencias, artículos de prensa o blogs especializados, etc.) utilizando las etiquetas propuestas: \#Relaciónate \#GradoRRLL y la propia de cada asignatura, tal como \#Sindical2 (resultado núm. 3); c) opinar y debatir en el hilo de mensajes de cada publicación (resultado núm. 4); d) plantear consultas al profesorado a través de mensajes realizados al hilo de los tweets publicados en la cuenta o mediante mensajes directos, ya se trate de conversaciones privadas como grupales entre las personas que forman la red creada (seguidores de la cuenta principal). Pero, asimismo, la información compartida en la red social ha propiciado que los estudiantes se mostraran más participativos en el aula, durante el desarrollo de las sesiones presenciales, aludiendo a cuestiones vistas en Twitter en numerosas ocasiones -ya fuera en la cuenta principal del proyecto, en la de compañeros o en otras afines, vinculadas al ámbito de las relaciones laborales-. 


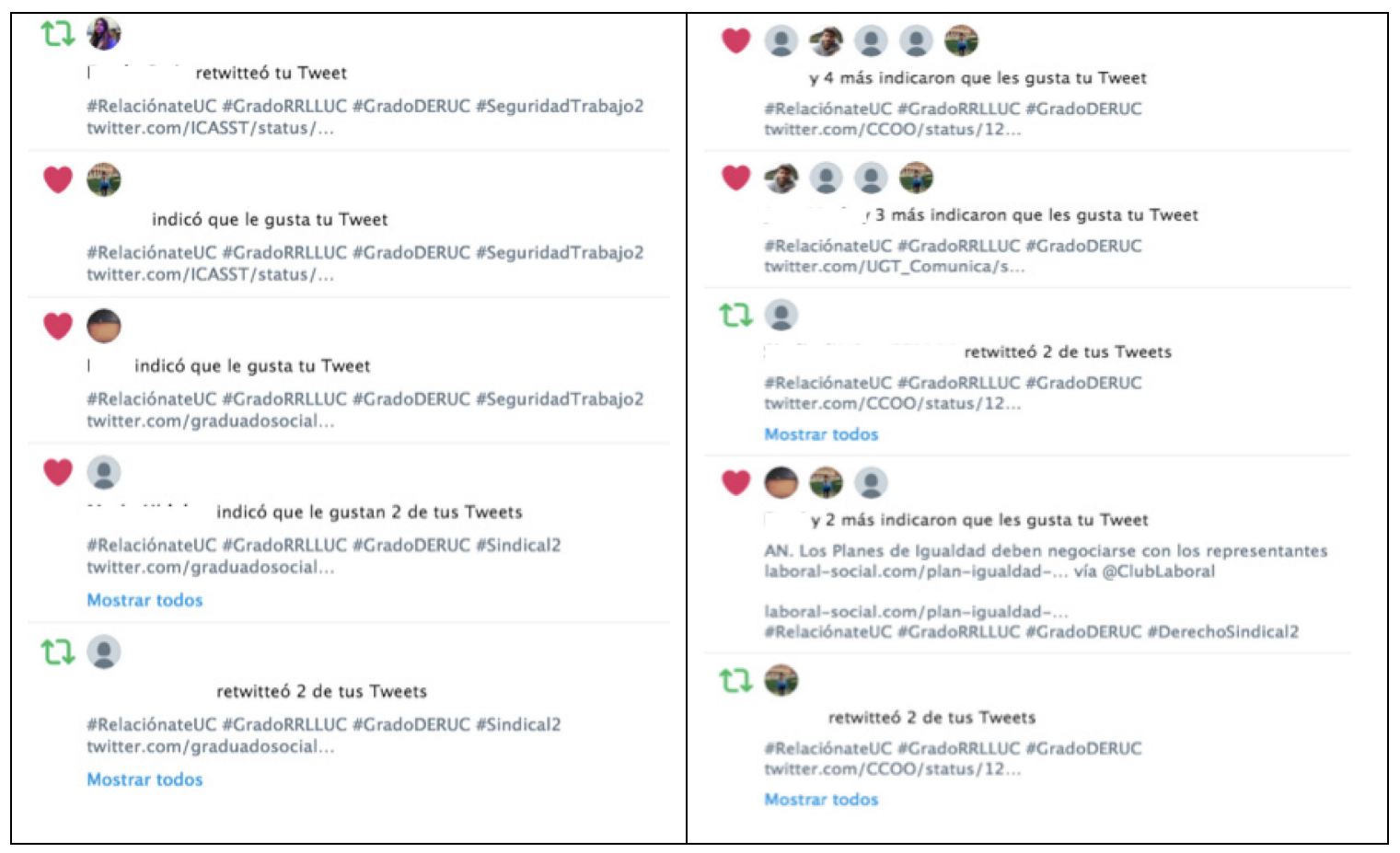

Resultados núm. 2 y 3: Interés mostrado por los estudiantes a las publicaciones de la cuenta principal mediante "me gusta" y retweets utilizando las etiquetas propuestas (selección).

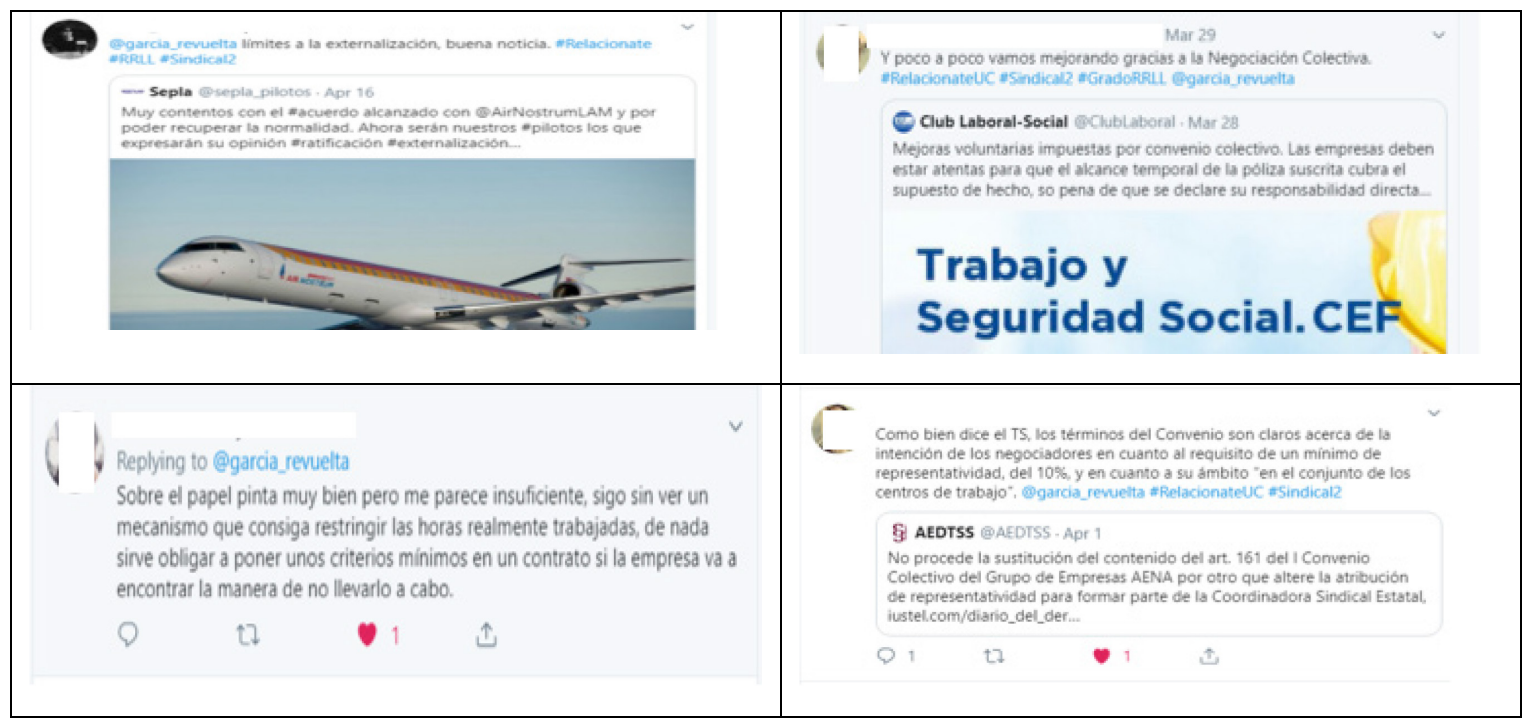

Resultado núm. 4: Publicaciones realizadas por los alumnos utilizando las etiquetas propuestas, incluyendo opiniones o valoraciones (selección).

La difusión de contenido a través de Twitter supone la selección de material docente que sirve como complemento a las explicaciones teóricas y prácticas realizadas por el profesor en el aula. De modo ejemplificativo, se ha compartido el "mapa de la negociación colectiva" elaborado por la Comisión Consultiva Nacional de Convenios Colectivos, como herramienta útil para que los estudiantes puedan identificar los convenios colectivos aplicables a los supuestos prácticos propuestos en la asignatura "Derecho Sindical II" (resultado núm. 5). O el texto de novedades normativas que han sido aprobadas con posterioridad a la explicación teórica de la materia en el aula, permitiendo a los alumnos mantenerse 
actualizados (resultado núm. 6). Pero también sentencias y comentarios doctrinales que servirán de base en el planteamiento de supuestos prácticos evaluables (resultado núm. 7). O noticias sobre la materia, enlaces a blogs especializados o a artículos doctrinales, etc. (resultado núm. 8).

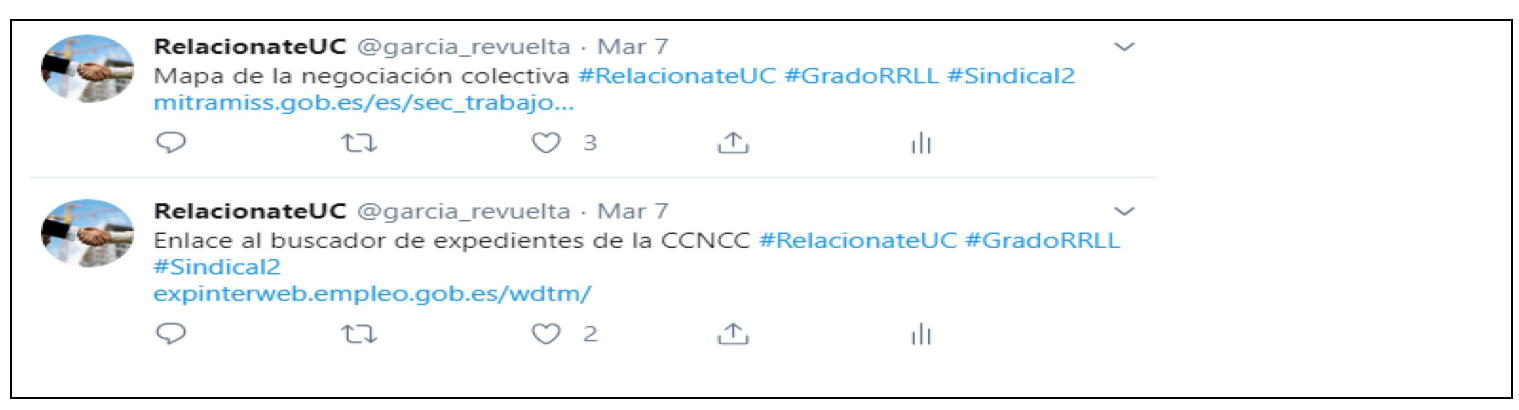

Resultado núm. 5: Publicación de herramientas útiles, tales como el mapa de la negociación colectiva o el buscador de expedientes de la Comisión Consultiva Nacional de Convenios Colectivos.

RelacionateUC@garcia_revuelta. Mar7

Publicado el Real Decreto Ley de medidas para la igualdad de trato y de

oportunidades en el empleo y la ocupación . Noticias Jurídicas

noticias.juridicas.com/\#.XIF8GzmR5Gk....

\#RelacionateUC \#GradoRRLL \#Sindical2

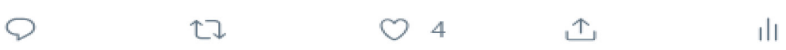

Resultado núm. 6: Publicación de novedades normativas.

En definitiva, frente al modelo tradicional basado en la impartición de clases magistrales con escasa o nula participación de los estudiantes, completadas con sesiones prácticas en la que el profesor plantea un caso de manera unilateral, el desarrollo de este proyecto ha permitido innovar en la metodología docente. De este modo, se ofrece a los alumnos información y contenidos formativos complementarios para el seguimiento de las clases teóricas y prácticas, que facilita que tengan un conocimiento previo y profundo sobre temas de actualidad, favoreciendo así su participación activa en el aula a través de intervenciones en las que aportan sus conocimientos y sus opiniones, lo que aumenta su implicación e interés.

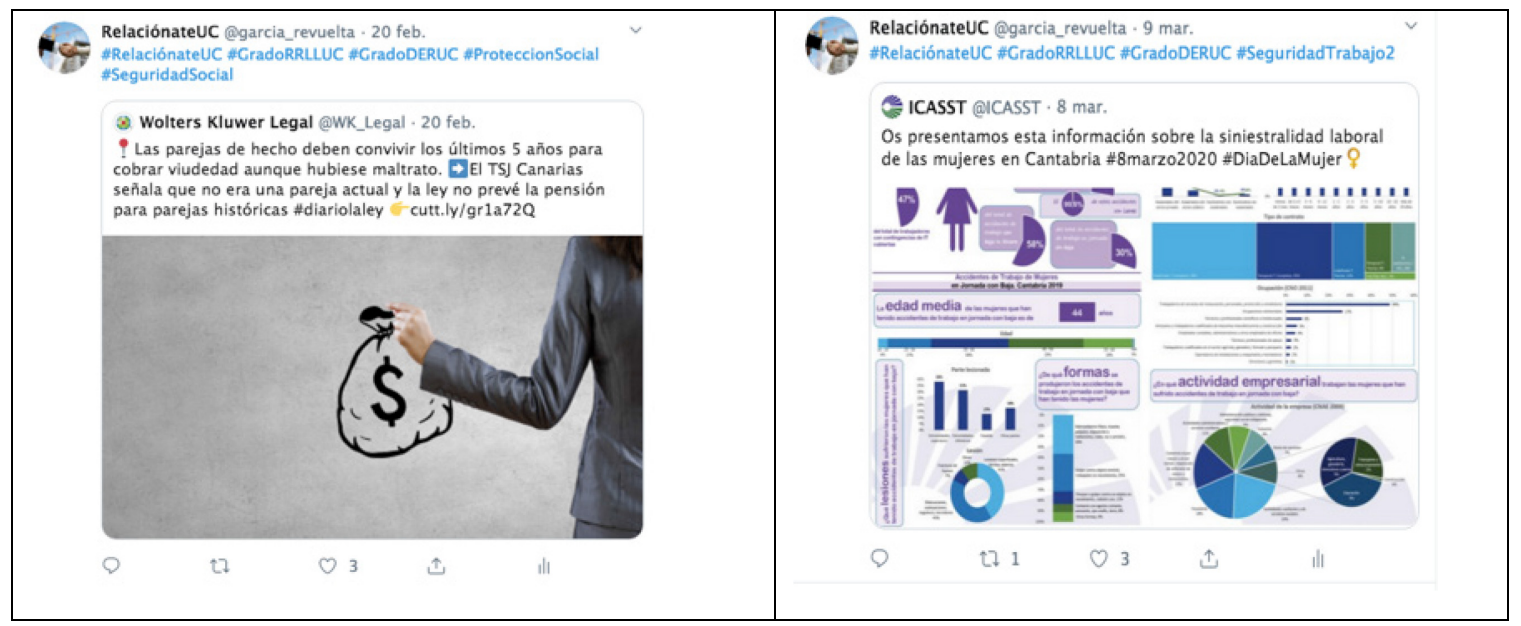

Resultados núm. 7 y 8: Publicación de sentencias, comentarios doctrinales, noticias de actualidad y demás información de interés.

(c) BY-NC-ND 2020, Universitat Politècnica de València

Congreso In-Red (2020) 


\subsection{Valoración de los estudiantes implicados}

Con la finalidad de conocer la opinión de los estudiantes sobre la utilidad del proyecto de innovación docente, al finalizar el curso 2018-2019 se puso a disposición de los matriculados en las asignaturas implicadas en esta actividad una encuesta de valoración. Las mismas preguntas han sido planteadas a los estudiantes durante el curso 2019-2020, con el objetivo de analizar aquellos aspectos a mejorar. Así, preguntados por las siguientes cuestiones, los alumnos encuestados responden:

\section{1. ¿Tienes cuenta de Twitter?}

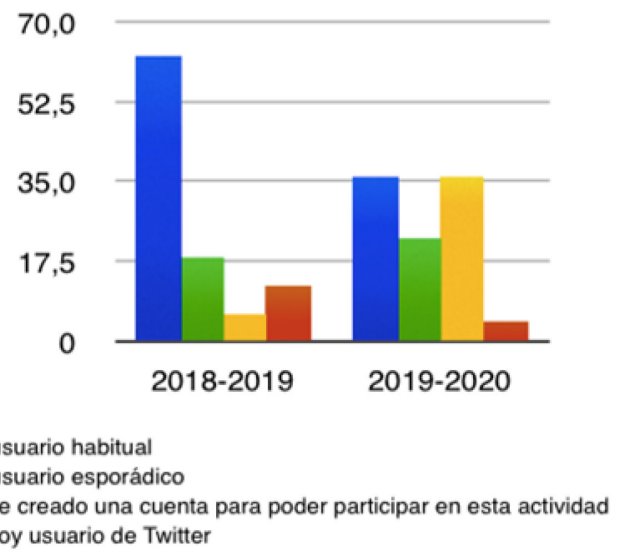

\section{2. ¿Qué tipo de perfil utilizas para hacer el seguimiento del proyecto}

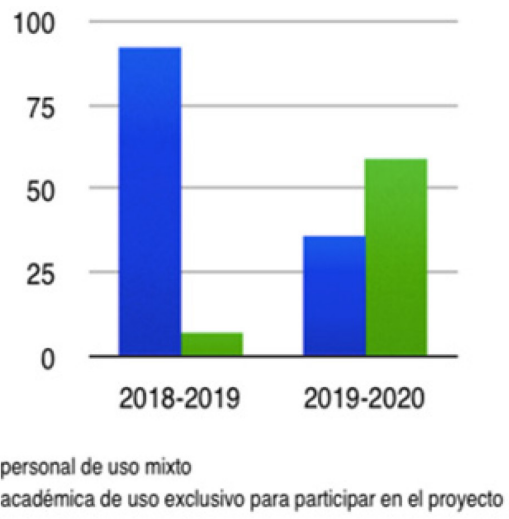

A la vista de las respuestas, resulta destacable el dato de que la mayoría de los estudiantes eran usuarios de Twitter con anterioridad pero que nunca habian utilizado esta red social con fines académicos (100\% curso 2018/2019; 100\% curso 2019/2020). 


\section{3. ¿Habías usado Twitter antes con fines académicos?}

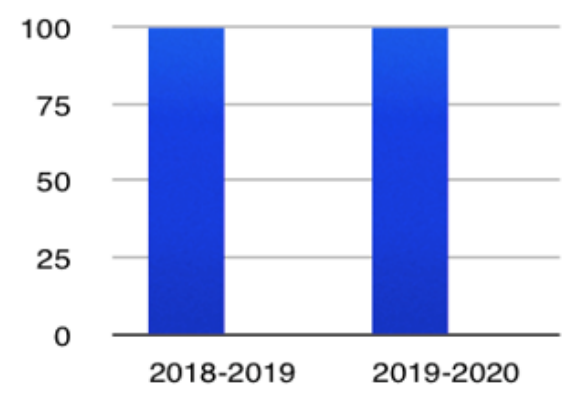

No, solo lo usaba en tiempo de ocio

Si, era seguidor de perfiles especializados en relaciones laborales

\section{4. ¿Te parece útil crear una red especializada en material de relaciones laborales?}

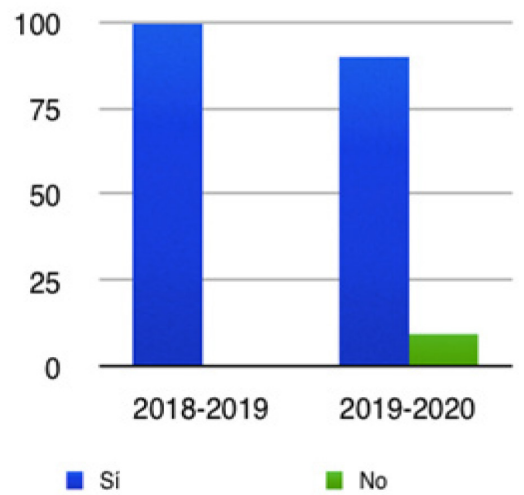

5. ¿Con qué frecuencia consultas el perfil@RelaciónateUC?

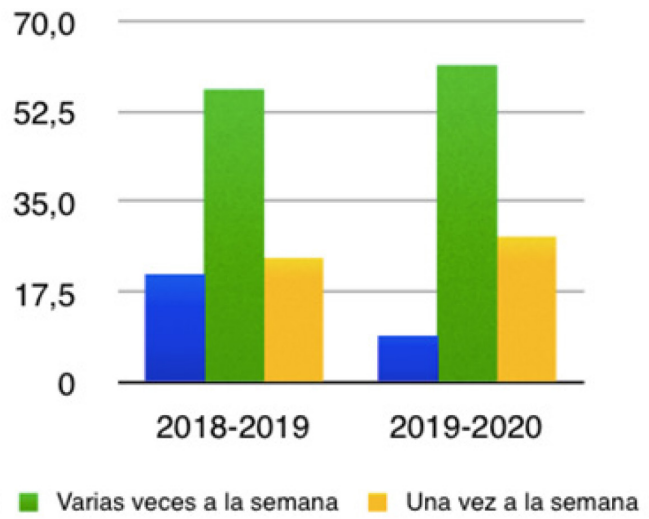

Asimismo, se aprecia un alto nivel de seguimiento pasivo: manifiestan consultar el perfil (a) Relaciónate varias veces por semana el 57,14\% en el curso 2018/2019 y el 61,90\% en el curso 2019/2020. 
Desarrollo de comunidades de aprendizaje en línea para titulaciones jurídicas. \#Relaciónate...

6. ¿Cuántas veces has dado a "me gusta" en una publicación vinculada al proyecto?

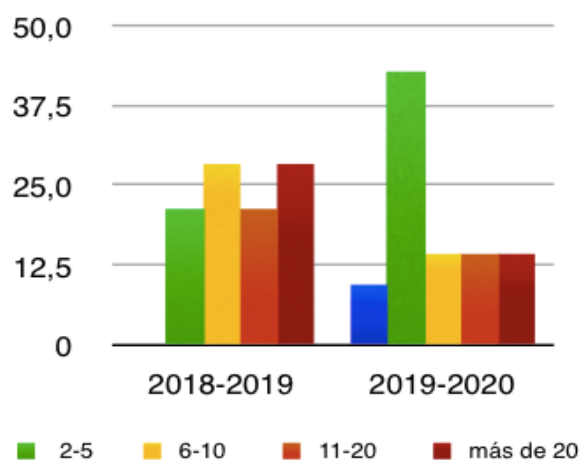

7. ¿Has compartido contenido relacionado con el proyecto?

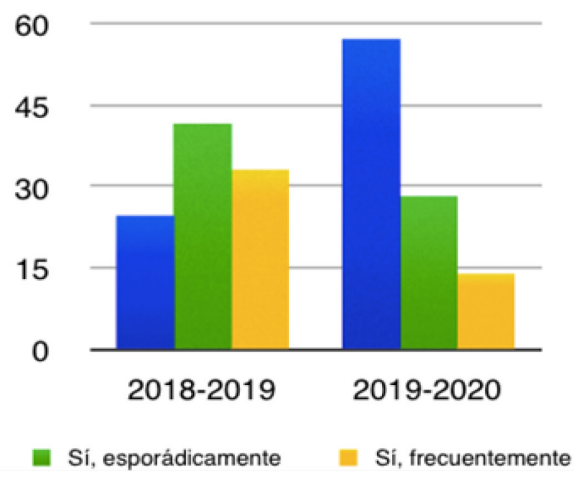

Si bien, la participación activa es minoritaria: el 25,00\% en 2018/2019 y el 57,14\% en 2019/2020 indica que no ha publicado nunca contenidos en el contexto del proyecto. Por lo que se entiende necesario reforzar este aspecto.

8. ¿Has publicado algún comentario en el contexto del proyecto?

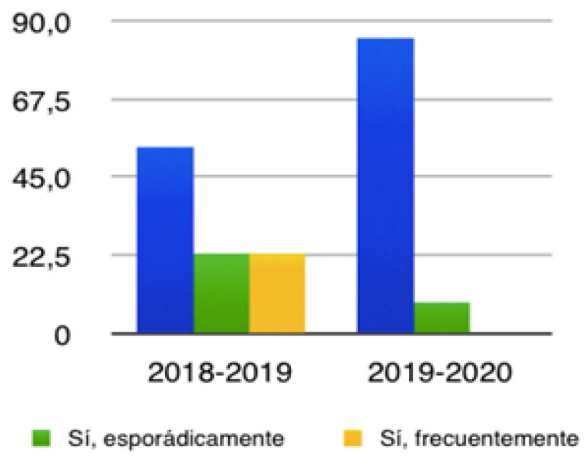




\section{9. ¿Te han resultado interesantes los contenidos publicados por las cuenta principal?}

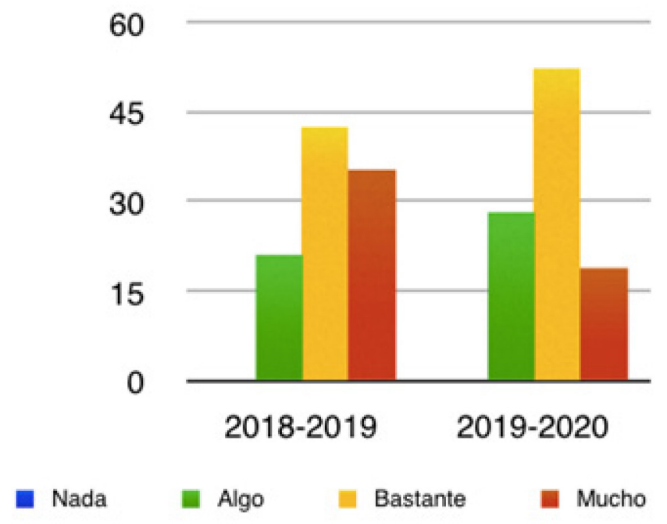

De forma mayoritaria consideran que los contenidos difundidos son interesantes: bastante-mucho $78,57 \%$ en $2018 / 2019$ y $71,46 \%$ en $2019 / 2020$.

\section{0. ¿El proyecto te parece útil para mejorar tus conocimientos?}

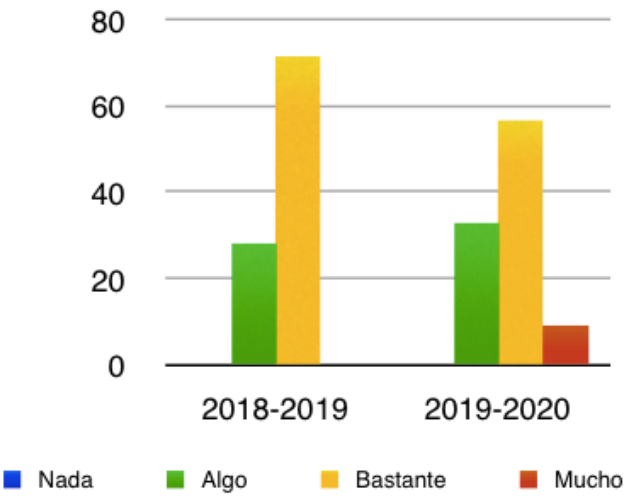

Y la valoración general de los estudiantes encuestados sobre el proyecto de innovación docente es muy positiva: bastante-mucho $71,43 \%$ en $2018 / 2019$ y 63,99\% en 2019/2020.

En conclusión, los alumnos se muestran familiarizados con la red social utilizada y atraidos por los contenidos publicados.Un alto porcentaje de los estudiantes involucrados ha realizado un seguimiento continuo de carácter pasivo. Y lo perciben como una actividad útil para su formación. Si bien, su participación activa (publicando, comentando, seleccionando contenidos) es minoritaria. Por tanto, las propuestas de mejora se orientan a la consecución del desarrollo de un papel más activo por parte de los alumnos. En este sentido, se considera necesario introducir incentivos relacionados con el sistema de evaluación continua, definiéndolo detalladamente en la guía docente. 


\section{Conclusiones y plan de futuro}

La estrategia de innovación docente implementada persigue favorecer un modelo de aprendizaje participativo, dinámico y colaborativo mediante el uso de los recursos que nos ofrecen las nuevas tecnologías de la comunicación. Inspirada en los principios de las metodologías activas, en las que, como es sabido, el estudiante es el protagonista y el profesor ejerce de orientador o guía, se ha creado un entorno de aprendizaje abierto y flexible, con el objetivo principal de potenciar la motivación, el interés y la implicación de los alumnos en su formación.

La plataforma Twitter, que permite la publicación de mensajes breves de manera sencilla, gratuita y versátil a través de diferentes formatos (texto, imágenes, vídeos, enlaces web, etc.), ha servido para crear un perfil (@RelaciónateUC) a través del cual difundir información y contenidos de utilidad e interés académico para los estudiantes del Grado en Relaciones Laborales y Derecho: noticias jurídicas, sentencias, análisis doctrinales, información disponible en sitios web de instituciones públicas o privadas, blogs especializados, etc. De este modo, contextualizar los contenidos teóricos y prácticos impartidos en el aula, dándose prioridad a los temas de máxima actualidad y mayor trascendencia social. Pero, asimismo, esta herramienta ha generado una red de comunicación y colaboración entre los estudiantes, que han mostrado interés por los materiales puestos a su disposición en la cuenta principal, han seleccionado aquellos de su preferencia, han compartido información con sus compañeros y han participado activamente ofreciendo sus opiniones y reflexiones en abierto y/o a través de conversaciones en modo privado, así como en los debates que los temas propuestos en la red social han suscitado en el aula.

De este modo, se considera que la actividad desarrollada en el marco de este proyecto contribuye adecuadamente a la consecución de los objetivos propuestos. Primero, permitiendo la difusión de conocimiento y de materiales docentes abiertos a través de las TIC, orientando a los alumnos en el acceso a información fiable y de calidad disponible en internet a través de la creación de una red de perfiles seleccionados para la finalidad descrita: organismos oficiales, editoriales jurídicas, medios de comunicación, perfiles de profesionales y especialistas, etc. Segundo, sirviendo como canal de comunicación abierto e inclusivo que propicia las relaciones entre estudiantes, así como entre alumnos y profesores, desarrollándose debates, intercambios de opiniones, compartiéndose información de interés, etc. Tercero, mejorando, de este modo, algunas competencias, habilidades y valores esenciales para los alumnos del Grado en Relaciones Laborales, tales como su capacidad de aprendizaje autónomo y su implicación en el proceso de formación académica; su razonamiento crítico y creativo; las habilidades de argumentación; la organización y gestión de información; el trabajo en equipo y la colaboración en la consecución de objetivos comunes.

Todo lo cual permite realizar una evaluación positiva de su impacto y nos compromete con su continuidad para lograr consolidar una comunidad de colaboración estable, abierta y transversal entre estudiantes, profesores, egresados y profesionales con un interés común, las relaciones laborales.

\section{Referencias bibliográficas}

Alegra Nueno, M., Esteve Segarra, A., López Balaguer, M., y Taléns Visconti, E., "Innovación docente en Derecho del Trabajo: propuestas prácticas” en CEF. Núm. 10. 2018.

Álvarez de Sotomayor, I. D., Tesis doctoral Posibilidades educativas de las redes sociales. Facultad de Ciencias de la Educación, Departamento de Pedagogía y Didáctica. Universidad de La Coruña. 2014. 
Arrabal Platero, P., "Capítulo 7. Nuevas metodologías docentes en el proceso enseñanza-aprendizaje semipresencial” en La docencia del Derecho en la sociedad digital. Huygens. Barcelona. 2019.

BLANCO GARCíA, A.I. "El uso de blogs en la innovación docente: un nuevo paradigma de enseñanzaaprendizaje" en Actualidad Jurídico Iberoamericana. 2016.

CANTÓ-LÓPEZ, M., "Introducing group work and profesional writing in a British Law School: a practical assignment and students' perceptions inside the liberal v vocational Law degree dichotomy" en REJIE. Revista Jurídica de Investigación e Innovación Educativa. Núm. 22. 2020.

FERNÁNDEZ-COCA, A., "El uso de sus redes 2.0 como herramienta de conquista del interés por la materia" en La Cuestión Universitaria. Monográfico Los estudiantes universitarios en la era digital. Núm.7. 2011.

GEWERC, A., Montero, L., Y LAMA, M., "Colaboración y redes sociales en la enseñanza universitaria” en Comunicar. Núm. 42. 2014.

GISBERT, M., Y EsteVE, F., "Digital Leaners: la competencia digital de los estudiantes universitarios" en La Cuestión Universitaria. Monográfico Los estudiantes universitarios en la era digital. Núm.7. 2011.

GÓMEZ, M., Roses, S., Y FARIAS, P., "El uso académico de las redes sociales en universitarios" en Comunicar. Núm. 38. 2012.

Holgado SÁez, C., "Las nuevas tecnologías en los estudios de Derecho en el marco del EEES: sugerencias didácticas de actividades colaborativas con entornos virtuales" en REJIE: Revista Jurídica de Investigación e Innovación Educativa. Núm. 3. 2011.

LARO GONZÁLEZ, E., "Innovar enseñando: la educación del futuro. Las TICs como factor motivador en la enseñanza” en REJIE. Revista Jurídica de Investigación e Innovación Educativa. Núm. 21. 2020.

MARTíneZ, A., FERnÁNDEZ, R., "Las redes sociales como espacios virtuales de aprendizaje” en Revista de Estudios e Investigación en Psicología y Educación. Núm. 13. 2017.

Nieto Rojas, P., "La utilización de Twitter como herramienta docente: su aplicación al grado de relaciones laborales y empleo" en Lan Harremanak Especial. 2017.

Periago Morant, J.J., "Capítulo 4. TICs y redes sociales en Derecho Penal: pensamiento analítico" en La docencia del Derecho en la sociedad digital. Huygens. Barcelona. 2019.

Quintero Lima, M.G., "La enseñanza del Derecho del Trabajo y de la Seguridad Social hoy" en REJIE: Revista Jurídica de Investigación e Innovación Educativa. Núm. 5. 2012.

Romero LóPez, S., Tesis doctoral Positive Reinforcement Social Networks: online social interactions to measure, analyse and improve academic outcomes. Universidad de Castilla-La Mancha. 2016.

Rosino CALle, R., y Álvarez RodríGuez, I., "Retos del presente y del futuro: la innovación docente en el ámbito del derecho" en Revista de Formación e Innovación Educativa Universitaria. Vol. 7. Núm. 3. 2014.

VÁzquez-Martínez, A.I., y CABero-Almenara, J., "Las redes sociales aplicadas a la formación" en Revista Complutense de Educación. Vol. 26. 2015. 